\title{
Kinetics of suicide substrates
}

\author{
Practical procedures for determining parameters
}

\author{
Stephen G. WALEY \\ Sir William Dunn School of Pathology, University of Oxford, South Parks Road, Oxford OX1 3RE, U.K.
}

\section{(Received 11 October 1984/Accepted 14 December 1984)}

\begin{abstract}
Many clinically important or mechanistically interesting inhibitors react with enzymes by a branched pathway in which inactivation of the enzyme and formation of product are competing reactions. The steady-state kinetics for this pathway [Waley (1980) Biochem. J. 185, 771-773] gave equations for progress curves that were cumbersome. A convenient linear plot is now described. The time $\left(t_{t}\right)$ for $50 \%$ inactivation of the enzyme (this is also the time for $50 \%$ formation of product), or for $50 \%$ loss of substrate, is measured in a series of experiments in which the concentration of inhibitor, $[\mathrm{I}]_{0}$, is varied; in these experiments the ratio of the concentration of enzyme to the concentration of inhibitor is kept fixed. Then a plot of $[\mathrm{I}]_{0} \cdot t_{1}$ against $[\mathrm{I}]_{0}$ is linear, and the kinetic parameters can be found from the slope and intercept. Furthermore, simplifications of the equations for progress curves are described that are valid when the concentration of inhibitors is high, or is low, or when the extent of reaction is low. The use of simulated data has shown that the recommended methods are not unduly sensitive to experimental error.
\end{abstract}

Suicide substrates, or mechanism-based inhibitors, may be defined in terms of their dual role as compounds that interact with enzymes by a mechanism with a branched pathway, the branches representing turnover of substrate and inactivation of enzyme: and little guidance that is a help in practice. Kinetic measurements are often sparse in papers on mechanism-based inhibitors, partly because theoretical treatments have not been developed to the point of usefulness. This is the gap that the present paper seeks to fill.

Suicide substrate + enzyme $\rightarrow$ complex $\mathrm{I} \rightarrow$ complex $\mathrm{II} \rightarrow$ product + enzyme (turnover)

$\downarrow$ (inactivation)

dead enzyme

There is now a wide range both of mechanismbased inhibitors (Walsh, 1982, 1984) and of enzymes involved; examples include a monoamine oxidase (Tipton et al., 1983), cytochrome $P-450$ (Ortiz de Montellano \& Mico, 1981), $\beta$-lactamases (e.g. Frère et al., 1982) and an aldolase (Meloche, 1981). Several mechanism-based inhibitors are used clinically, e.g. clavulanic acid and 5-fluorouracil in the treatment of bacterial infections and neoplastic diseases respectively. Mechanismbased inhibitors appeal to the medicinal chemist because of their potential for selective action in vivo, and are useful to enzymologists studying active sites. Despite the importance of suicide substrates, there have been few detailed studies of their kinetics (Waley, 1980; Tatsunami et al., 1981)
The dual role of these compounds as substrates and inhibitors means that two types of work need to be considered in testing models. In one type, the compound is regarded primarily as a substrate, and the main question is whether there is some inactivation of the enzyme or not. In the other type of work the compound is regarded primarily as an inhibitor, and the question is whether there is some turnover or not. Tests for inactivation are considered later below. A test for turnover, and in fact an important first step in all quantitative work with these compounds, is to determine the molar proportion for inactivation, i.e the number of molecules of inhibitor required to inactivate one molecule of enzyme.

The mechanism is that considered previously 
(Waley, 1980); the symbol for the compound (suicide substrate or mechanism-based inhibitor) is now I:

$$
\mathrm{E}+\mathrm{I} \stackrel{k_{+1}}{\stackrel{k_{-1}}{\rightleftharpoons}} \mathrm{X} \stackrel{k_{+2}}{\longrightarrow} \mathrm{Y} \stackrel{k_{+3}}{\longrightarrow} \mathrm{E}+\mathrm{P}
$$

$E$ and $E_{i}$ are enzyme and inactivated enzyme respectively, $P$ is product, $X$ is the first intermediate (the enzyme-substrate complex) and $Y$ is another intermediate (e.g. an acyl-enzyme). The intermediate $Y$ has a choice of reaction, governed by the partition ratio $r$, where $r=k_{+3} / k_{+4}$. The molar proportion for inactivation, as defined above, may be determined by plotting the fractional activity remaining against the ratio of the initial concentration of inhibitor to that of enzyme. The intercept on the abscissa is $1+r$ (see, e.g., Fig. 1 of Knight \& Waley, 1985). The constancy of the partition ratio when the concentrations of inhibitor (substrate) and enzyme are varied is a useful test for this mechanism.

The basic features of the steady-state solution are now outlined. Then inactivation is described, first alone, and then in the presence of monitoring substrate. Next, the equations for the course of turnover are given, and finally the pre-steady-state solution is mentioned. Convenient graphical methods for determining the kinetic parameters are described. The essence of the recommended procedure can be gleaned from Fig. 1 and Table 2 . Advice on the use of progress curves has been given by Wharton \& Szawelski (1982).

\section{Theory}

\section{Basic features of the kinetic scheme}

The competing pathways in the kinetic scheme have the consequence that $p / e_{\mathrm{i}}=r$, where $p$ and $e_{\mathrm{i}}$ are the concentrations of $P$ and $E_{i}$. The outcome of an experiment depends on the balance between the relative concentrations of enzyme and substrate, and the value of $r$. If the initial concentrations of inhibitor and enzyme are $[\mathrm{I}]_{0}$ and $e_{0}$ respectively, then the enzyme is completely inactivated when $[\mathrm{I}]_{0}$ is greater than $(1+r) e_{0}$, the final concentration of product being $r \cdot e_{0}$. On the other hand, if $[\mathrm{I}]_{0}<(1+r) e_{0}$, then the outcome is that all of the substrate is consumed, the final enzymic activity being $e_{0}-[\mathrm{I}]_{0} /(1+r)$, and the final concentration of product being $r[\mathrm{I}]_{0} /(1+r)$. It is convenient to define:

$$
M=(1+r) \cdot \frac{e_{0}}{[\mathrm{I}]_{0}}
$$

Thus the outcome is decided by whether $M<1$ (complete inactivation) or $M>1$ (complete consumption of substrate). Experimentally, there are three options for measuring the progress of the reaction: consumption of substrate, appearance of product and inactivation of the enzyme. A characteristic of the steady-state branched-pathway kinetics, not shared by the simpler kinetics of an unbranched (one substrate) pathway, is that the rates of consumption of substrate and appearance of product differ; the difference will be small when $e_{0} \ll[\mathrm{I}]_{0}$.

The kinetic parameters that can be obtained from steady-state progress curves are the firstorder rate constant for inactivation $\left(k_{\text {in }}\right)$ (that for turnover being $r \cdot k_{\text {in }}$ ), and $K^{\prime}$, the counterpart of a Michaelis constant (Table 1). The parameter $r$ is best obtained from the final extent of partial inactivation.

\section{Course of inactivation}

This section gives equations that are convenient to use when it is the inactivation of the enzyme that is being measured. The cumbersome equations deduced previously (Waley, 1980; Tatsunami et al., 1981) can be simplified. Thus the progress curve for the relative concentration of inactivated enzyme can be expressed as

$$
t=N \cdot \ln (1-M \cdot z)-N^{\prime} \cdot \ln (1-z)
$$

where $z=e_{\mathrm{i}} / e_{0}$ and $N$ and $N^{\prime}$ are defined in Table 1. I now describe ways of obtaining values of the kinetic parameters from experimental data. Tests showed that multiple linear regression was unduly sensitive to errors: e.g. a standard deviation of 0.0025 (see the Appendix) gave errors of about 10 $20 \%$ in $k_{\text {in }}$ and $K^{\prime}$. Discontinuous assays of the enzymic activity at various times would seldom be precise enough for multiple linear regression to be useful. However, the $t_{t}$ plot now described is adequately robust. The time for $50 \%$ inactivation $\left(t_{i}\right)$ is readily obtained experimentally by interpolation (Fig. 1), and when $z=0.5$ eqn. (2) may be rearranged and the values for $N$ and $N^{\prime}$ substituted to give:

$$
[\mathrm{I}]_{0} \cdot t_{\frac{1}{2}}=\left(\frac{\ln (2-M)}{1-M}\right) \frac{K^{\prime}}{k_{\text {in }}}+\frac{\ln 2}{k_{\text {in }}} \cdot[\mathrm{I}]_{0}
$$

Then $[\mathrm{I}]_{0} \cdot t_{4}$ is plotted against $[\mathrm{I}]_{0}$ for a series of experiments in which $[\mathrm{I}]_{0}$ is varied but the quotient $e_{0} /[\mathrm{I}]_{0}$ is kept fixed. The kinetic parameters $k_{\text {in }}$ and $K^{\prime}$ are found from the slope and intercept (Table 2). When the standard deviation (see the Appendix) was 0.01 , corresponding to $2 \%$ at $50 \%$ inactivation, the value of $t_{4}$ found was unbiassed and the coefficient of variation (from 11 simulated experiments) was $2 \%$. The parameters were found to 
Table 1. Symbols, definitions and relationships for the kinetics of suicide substrates

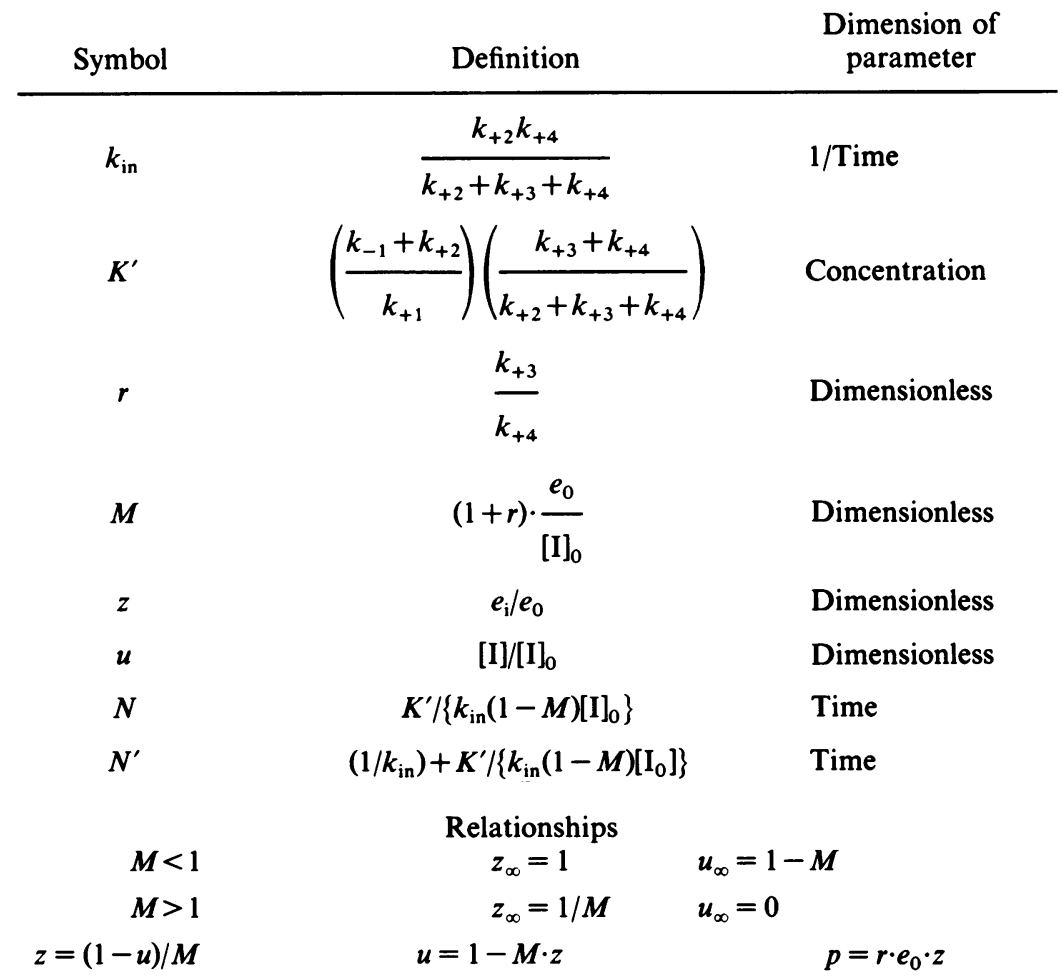

Table 2. Kinetic parameters from $t_{4}$ plots

The time at which there is $50 \%$ inactivation of the enzyme, or $50 \%$ formation of product in turnover, is $t_{\frac{1}{t}}$ in the first section of the Table; $t_{4}$ is $50 \%$ loss of substrate in the second section. The initial concentration of mechanism-based inhibitor or suicide substrate is $[\mathrm{I}]_{0}$ and of enzyme $e_{0}$. The quotient $e_{0} /[\mathrm{I}]_{0}$ is kept fixed and $[\mathrm{I}]_{0}$ varied in a series of experiments in which $t_{\xi}$ is measured, and then $[\mathrm{I}]_{0} \cdot t_{\xi}$ is plotted against $[\mathrm{I}]_{0}$. The kinetic parameters $k_{\text {in }}$ (units, say, $\min ^{-1}$ ) and $K^{\prime}$ (units, say, $\mu \mathrm{M}$ ) are found from the formulae given opposite them; $M=(1+r) e_{0} /[1]_{0}$, where $1+r$ is the number of turnovers that inactivates the enzyme.

Inactivation of enzyme or formation of product measured

$$
\begin{array}{cc}
k_{\text {in }} & \frac{\ln 2}{\text { Slope }} \\
K^{\prime} \quad\left(\text { Ordinate intercept) } \cdot k_{\text {in }} \cdot \frac{1-M}{\ln (2-M)}\right. \\
\text { Loss of suicide substrate measured } \\
k_{\text {in }} & \frac{\ln \left(\frac{M}{M-0.5}\right)}{\text { Slope }} \\
K^{\prime} \quad(\text { Ordinate intercept }) \cdot k_{\text {in }} \cdot \frac{1-M}{\ln \left(\frac{M}{2 M-1}\right)}
\end{array}
$$

Vol. 227 

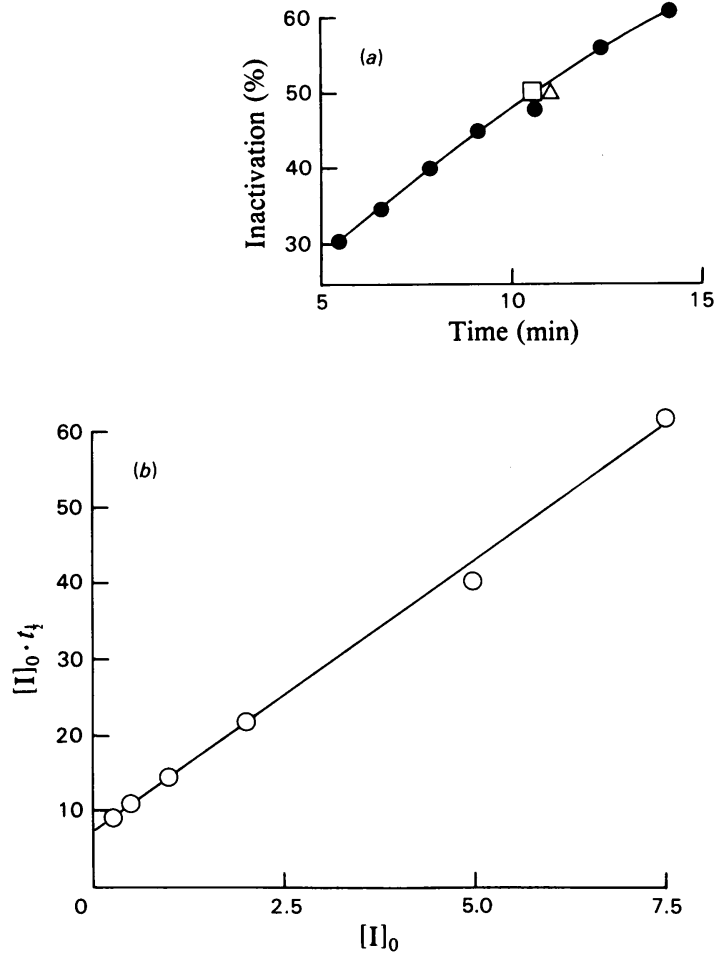

Fig. 1. Half-time plot for mechanism-based inhibitors (a) Variation of percentage inactivation with time of reaction; $e_{0}=0.004,[\mathrm{I}]_{0}=2, \quad r=100, \quad K^{\prime}=1$, $k_{\text {in }}=0.1$. Simulated experimental points; - , error-free curve; $\square$, error-free $t_{\mathrm{t}}$. The two points each side of $50 \%$ inactivation were used for interpolation and gave the simulated experimental $t_{t}$ $(\triangle)$ of $11 \mathrm{~min}$. (b) The plot of $[\mathrm{I}]_{0} \cdot t_{t}$ against $[\mathrm{I}]_{0}$ for a series of experiments in which $[\mathrm{I}]_{0}$ was varied from 0.25 to 7.5 and $e_{0} /[\mathrm{I}]_{0}$ was kept fixed at 0.002 . The theoretical values of $k_{\text {in }}$ and $K^{\prime}$ were 0.1 and 1 respectively, and the found values were 0.097 and 0.96. The simulated experimental error had standard deviation 0.01 in both $(a)$ and $(b)$.

about $5 \%$ in the set shown in Fig. 1. Thus this is the preferred general method.

The effects of the values of $M$ and of $[\mathrm{I}]_{0} / K^{\prime}$ are now considered. The extent of inactivation attains $50 \%$ when $M<0.5$. When $M \ll 1$, the first term on the right-hand side of eqn. (3) in large parentheses reduces to $\ln 2$, and the course of inactivation is first-order. That there might be only slight deviation from linearity in the usual semi-logarithmic plot of activity against time has been pointed out (Waley, 1980); $M$ was 0.1 or 0.02 in the example illustrated. Loosely speaking, inactivation always starts first-order, and, at sufficiently high concentrations of inhibitor, inactivation remains firstorder for as long as $[\mathrm{I}] \gg K^{\prime}$. On the other hand, when $[\mathrm{I}]_{0} \ll K^{\prime}$ then it may be shown that eqn. (2)

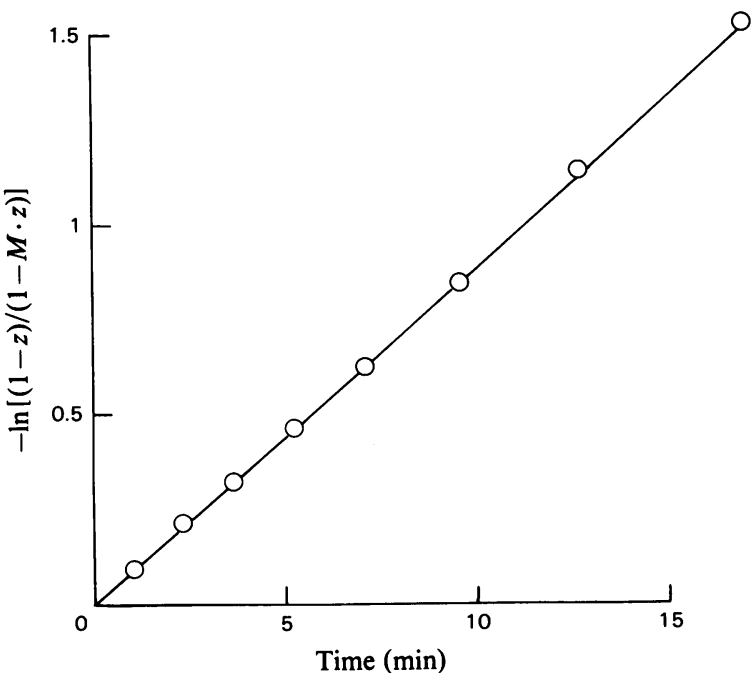

Fig. 2. Semi-logarithmic plot for inactivation with low concentration of inhibitor

Plot of $-\ln [(1-z) /(1-M \cdot z)]$ against time, an approximation valid for $[\mathrm{I}]_{0} \ll K^{\prime}$. Simulated data, standard deviation 0.01 . The concentrations of enzyme and substrate were $10^{-5}$ and $10^{-2}$ respectively; $\quad k_{\text {in }}=10 \mathrm{~min}^{-1}, \quad K^{\prime}=1, \quad r=100 \quad(\therefore$ $M=0.101)$. The slope of the line gives $k_{\mathrm{in}} / K^{\prime}=9.8$, obtained from:

$$
\frac{k_{\text {in }}}{K^{\prime}}=\frac{\text { slope }}{\left(1-M[\mathrm{I}]_{0}\right.}
$$

reduces to:

$$
t=-N \cdot \ln \left(\frac{1-z}{1-M \cdot z}\right)
$$

An example of the corresponding semi-logarithmic plot is shown (Fig. 2); the kinetic parameter found is the second-order rate constant, $k_{\text {in }} / K^{\prime}$, which is found from the slope.

Course of inactivation measured from progress curve of monitoring substrate

Often it is convenient to measure inactivation of the enzyme by comparison of the progress curves for a monitoring substrate alone and in the presence of the mechanism-based inhibitor. The rates of the reactions may be obtained (see the Appendix) and hence the time $\left(t_{1}\right)$ at which the rate is half that of the corresponding control reaction. A series of experiments with constant $e_{0} /[\mathrm{I}]_{0}$ are used as described above; values of $k_{\text {in }}$ and the apparent $K^{\prime}\left(K_{\text {app. }}^{\prime}\right)$ are obtained. Then from

$$
K_{\mathrm{app} .}^{\prime}=K^{\prime}\left(1+\frac{[\mathrm{S}]_{0}}{K_{\mathrm{m}}}\right)
$$

$K^{\prime}$ is found; here $[S]_{0}$ is the initial concentration of monitoring substrate whose Michaelis constant is 
$K_{\mathrm{m}}$. The concentration of monitoring substrate is chosen so that its value is not less than $60 \%$ of the original value, $[S]_{0}$, by the time that the rate of the inhibited reaction has declined to $40 \%$ of the control rate. The depletion of monitoring substrate may be allowed for by replacing $[S]_{0}$ in eqn. (5) by $[\overline{\mathrm{S}}]$, the mean concentration (cf. Lee \& Wilson, 1971).

\section{Progress curves for conversion of suicide substrate into product}

If the turnover of the suicide substrate is being measured then the $t_{4}$ plots differ according to whether formation of product or disappearance of substrate is being measured. Since the concentration of product $(p)$ is given by $p=r \cdot e_{0} z$, substitution in eqn. (2) gives:

$$
t=N \cdot \ln \left[1-M\left(\frac{p}{r \cdot e_{0}}\right)\right]-N^{\prime} \cdot \ln \left(1-\frac{p}{r \cdot e_{0}}\right)
$$

Then the $t_{4}$ equation for $50 \%$ formation of product is eqn. (3). If the disappearance of substrate is being measured, then it may be shown that:

$$
t=N \cdot \ln (u)-N^{\prime} \cdot \ln [(u+M-1) / M]
$$

where $u=[\mathrm{I}] /[\mathrm{I}]_{0}$.

The $t_{\sharp}$ method gives:

$[\mathrm{I}]_{0} \cdot t_{\frac{1}{2}}=\left(\frac{\ln \left(\frac{M}{2 M-1}\right)}{1-M}\right) \cdot \frac{K^{\prime}}{k_{\text {in }}}+\frac{\ln \left(\frac{M}{M-0.5}\right)}{k_{\text {in }}} \cdot[\mathrm{I}]_{0}$

Tests showed that the linear plot based on eqn. (8) was satisfactory: with a standard deviation of 0.01 in the relative substrate concentration $(u)$, the values of the kinetic parameters (see Table 2 ) were within $6 \%$ of their true values. Data conforming to the integrated Michaelis-Menten equation will also give a line when plotted according to eqn. (8), but the dependence of the slope and intercept on $[\mathrm{S}]_{0} / e_{0}$ is different.

The special cases of high and low concentrations of the suicide substrate parallel those treated above. Turnover remains first-order as long as $[\mathrm{I}] \gg K^{\prime}$, and conversely when $[\mathrm{I}]_{0} \ll K^{\prime}$ then:

$$
t=-N \cdot \ln \left(\frac{M-1+u}{M \cdot u}\right)
$$

\section{Detection of loss of enzymic activity during turnover}

So far I have assumed that the compound is known to be a suicide substrate or mechanismbased inhibitor. Since the rate of an enzymic reaction decreases owing to depletion of the substrate, it is not always easy to detect additional slowing down due to inactivation of the enzyme. Thus we now consider the problem of finding out whether a substrate participates in the branchedpathway inactivation. The useful linear $t_{t}$ plot described by Wharton \& Szawelski (1982), based on the integrated Michaelis-Menten equation, turned out not to be sensitive to partial inactivation of the enzyme by the present mechanism. Thus a simulated experiment in which $40 \%$ of the enzyme was inactivated at the end gave an acceptable line (Fig. 3). The residuals were scattered about their mean; although they all have the same sign, this, of course, would not be obvious in practice. Inactivation was, however, revealed by Selwyn's (1965) test (Fig. 3), a test also recommended by Wharton \& Szawelski (1982).

\section{Pre-steady-state kinetics}

The equations for the rates of change of the different species given earlier (Waley, 1980) may readily be solved with the Laplace-Carson transformation (see, e.g., Rodiguin \& Rodiguina, 1964). The solution for the fractional concentration of inactive enzyme, on the assumption that the concentration of free enzyme is low and that enzyme-substrate combination is rapid, is:

$$
z=1-\frac{a_{1} a_{2}}{a_{1}\left(a_{2}-a_{1}\right)} \cdot \mathrm{e}^{-a_{1} t}-\frac{a_{1} a_{2}}{a_{2}\left(a_{1}-a_{2}\right)} \cdot \mathrm{e}^{-a_{2} t}
$$

where

$$
\begin{aligned}
a_{1} a_{2} & =k_{+2} k_{+4} \\
a_{1}+a_{2} & =k_{2}+k_{+3}+k_{+4}
\end{aligned}
$$

Hence (see Table 1):

$$
k_{\text {in }}=a_{1} a_{2} /\left(a_{1}+a_{2}\right)
$$

If $a_{1} \gg a_{2}$ then, to a first approximation, $a_{1}=k_{+2}+k_{+3}+k_{+4}$ and $a_{2}=k_{\text {in }}$; moreover, the steady-state solution $z=1-\exp \left(-k_{\mathrm{in}} \cdot t\right)$ is valid at times much greater than $\left(k_{+2}+k_{+3}+k_{+4}\right)^{-1}$.

\section{Conclusions}

The improved procedures described should encourage the quantitative characterization of mechanism-based inhibitors. The significance of the kinetic parameters is now briefly discussed. The first-order constant $k_{\text {in }}$ is the catalytic constant for inactivation (cf. $k_{\text {cat }}$ ); if $k_{+2} \gg k_{+3}+k_{+4}$, then $k_{\text {in }}=k_{+4}$, the rate constant for the step leading to inactive enzyme. The constant $K^{\prime}$ is an analogue of a dissociation constant; thus (italic lower-case symbols denote concentrations), it may be shown that the dissociation constant $\left(K_{\mathrm{s}}\right)$ for the enzymesubstrate complex $(\mathrm{X})$, and $K^{\prime}$, are:

$$
K_{\mathrm{s}}=\frac{e \cdot s}{x} \quad \text { and } \quad K^{\prime}=\frac{e \cdot s}{x+y}
$$



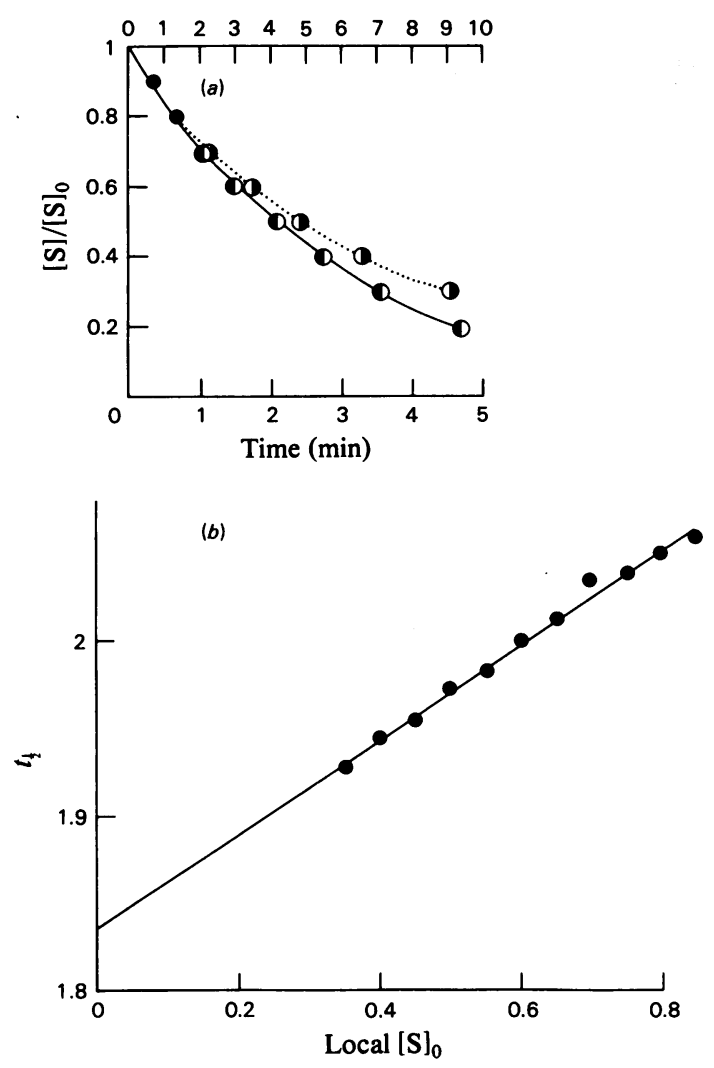

Fig. 3. Detection of enzyme inactivation Plot of $t_{\underline{t}}$ (ordinate) against local $s_{0}$ (abscissa) according to the Wharton \& Szawelski (1982) procedure for the reaction of a suicide substrate. The time was calculated from $t=N \cdot \ln (u)-N^{\prime}$. $\ln [(u+M-1) / M)]$, and noise with zero mean and standard deviation 0.005 was added; $u=[\mathrm{S}] /[\mathrm{S}]_{0}, N$ and $N^{\prime}$ are defined in Table 1. [S $]_{0}=1, e_{0}=0.05$, $K^{\prime}=1, r=50, k_{\text {in }}=0.25 \mathrm{~min}^{+1}$; thus $M=2.55$. The values of $t_{1}$ and local [S$]_{0}$ were then calculated from the simulated progress curve: the deviation from linearity is not obvious. The inset shows progress curves, plotted according to the Selwyn (1965) procedure. The lower (D) was calculated with the values given above and the time is given on the lower time scale, and the upper (o) was for $e_{0}=0.025$ and the time is given on the upper scale. The non-coincidence of the two curves shows inactivation of the enzyme.

If a series of mechanism-based inhibitors is being studied, an adequate understanding of structure-activity relationships demands knownledge of $k_{\text {in }}$ and $K^{\prime}$, as well as $r$, which measures the relative commitment to turnover.

The support of the Medical Research Council is gratefully acknowledged. This is a contribution from the Oxford Enzyme Group.

\section{References}

Frère, J. M., Dormans, C., Lenzini, V. M. \& Duyckaerts, C. (1982) Biochem. J. 207, 429-436

Knight, G. C. \& Waley, S. G. (1985) Biochem. J. 225, 435-439

Lee, Y.-J. \& Wilson, I. B. (1971) Biochim. Biophys. Acta 242, 519-522

Meloche, H. P. (1981) Trends Biochem. Sci. 6, 38-41

Ortiz de Montellano, P. R. \& Mico, B. A. (1981) Arch. Biochem. Biophys. 206, 43-50

Rodiguin, N. M. \& Rodiguina, E. N. (1964) Consecutive Chemical Reactions, pp. 111-131, Van Nostrand, Princeton

Selwyn, M. J. (1965) Biochim. Biophys. Acta 105, 193-195

Tatsunami, S., Yago, N. \& Hosoe, M. (1981) Biochim. Biophys. Acta 662, 226-235

Tipton, K. F., Fowler, C. J., McCrodden, J. M. \& Strolin Benedetti, M. (1983) Biochem. J. 209, 235-242

Waley, S. G. (1980) Biochem. J. 185, 771-773 (correction $1982,203,807)$

Walsh, C. (1982) Tetrahedron 38, 871-909

Walsh, C. (1984) Annu. Rev. Biochem. 53, 493-535

Wharton, C. W. \& Szawelski, R. J. (1982) Biochem. J. 203, 351-360

\section{APPENDIX}

\section{Simulated experiments}

The effect of error on the methods for obtaining parameters was studied as follows. An 'error-free' set of values for the time $(t)$ was calculated from the equations [e.g. eqn. (2) in the text] with $k_{\text {in }}$ $=0.1 \mathrm{~min}^{-1}$ and $K^{\prime}=1$ (concentration units are omitted for brevity). Then, to each value of $z$ (or $u$ ), one of a set of normally distributed pseudo-random numbers was added; these had mean zero, and standard deviation as chosen (often 0.01 ), and were obtained from the BASIC RND function of a Research Machines Ltd. microcomputer. The error-free time and the 'noisy' $z$ (or $u$ ) data were then used as simulated experimental data. The $t_{t}$ was found as the value of $t$ for which $z$ (or $u)=0.5$, by linear inverse interpolation (see, e.g., Hamming, 1971). Then $[\mathrm{I}]_{0} \cdot t_{4}$ was plotted against $[\mathrm{I}]_{0}$ and the values of $k_{\text {in }}$ and $K^{\prime}$ were thus obtained.

\section{Progress curve of monitoring substrate}

The rate of formation of product (Q) from monitoring substrate when the enzyme is losing activity by reaction with a mechanism-based inhibitor is given by:

$$
\frac{\mathrm{d} Q}{\mathrm{~d} t}=C(1-z)
$$

where $Q$ is written for [Q], $C$ is the control rate in the absence of inhibitor, and $z$ is the fractional concentration of inactive enzyme, as in the main 
text. When $z=0.5$, the rate is half the control rate. Thus the simplest procedure in practice is to measure the rate at different times and interpolate to obtain $t_{t}$, the time at which the rate is half the control rate. If constant time intervals $(h)$ are used, then $\left(Q_{i+1}-Q_{i-1}\right) / 2 h$ is an approximation to the rate at $Q_{\mathrm{i}}, t_{\mathrm{i}}$. Alternatively, and preferably, use of a parabola to smooth enables the rate at time $t_{\mathrm{i}}$ to be found from five points, the two points before and after $Q_{\mathrm{i}}, t_{\mathrm{i}}$ :

$$
\text { Rate }=\left(-2 Q_{i-2}-Q_{i-1}+Q_{i+1}+2 Q_{i+2}\right) /(10 h)
$$

Use of the progress curve (rather than the rate) is less simple, except under the conditions now described. The progress curve is given by:

$$
Q=C \int_{0}^{t}(1-z) \cdot \mathrm{d} t
$$

With the use of eqn. (2) in the text it may be shown that the solution is:

$$
Q=C\left(\frac{1}{k_{\mathrm{in}}} \cdot z-\frac{K_{\mathrm{app} .}^{\prime}}{k_{\mathrm{in}} \cdot M} \cdot \frac{1}{[\mathrm{I}]_{0}} \cdot \ln (1-M \cdot z)\right.
$$

This equation, together with eqn. (2) in the main text, defines the progress curve, but does not give $Q$ as an explicit function of time. However, when $M \cdot z \ll 1$, the logarithmic term may be expanded, and then:

$$
Q=\frac{C}{k_{\text {obs. }}}\left(1-\mathrm{e}^{-k_{\text {obs. }} \cdot t}\right)
$$

where

$$
k_{\text {obs. }}=\frac{k_{\text {in }}[\mathrm{I}]_{0}}{[\mathrm{I}]_{0}+K_{\text {app. }}^{\prime}}
$$

In this limiting case the progress curve is a simple exponential one. As a guide, when $M \leqslant 0.05$, eqn. (2) will be approximately valid for the first $60 \%$ of reaction.

\section{Reference}

Hamming, R. W. (1971) Introduction to Applied Numerical Analysis, pp. 143-145, McGraw-Hill Kogakusha, Tokyo 